\title{
Sublimable materials facilitate the TEM sample preparation of oil-soluble nanomaterials
}

\author{
Yu-Hao Denge
}

\begin{abstract}
Sample preparation is significantly important to the high-resolution transmission electron microscopy (HRTEM) characterization of nanomaterials. However, many general organic solvents can dissolve the necessary organic polymer support layer in TEM grid, which causes it difficult to obtain high-quality samples of oil-soluble nanomaterials. In this study, a new sample preparation method for oil-soluble nanomaterials has been developed by using the sublimable material as a transition layer. Experiments also show that there is no damage to TEM grids and high-quality HRTEM images can be obtained via this method. This approach paves the way to applicable HRTE M sample preparation of oil-soluble nanomaterials.
\end{abstract}

Keywords: Transmission electron microscopy, Sample preparation, Oil-soluble nanomaterial, Sublimable material

\section{Introduction}

Sample preparation is significantly important to the transmission electron microscopy (TEM) characterization for the accurate morphology and crystal structure of nanomaterials (Ayache et al. 2010; Cha et al. 2016; Kim et al. 2014; Park et al. 2016). In order to obtain high-quality high-resolution TEM (HRTEM) images, nanomaterials are often dispersed on the TEM grid, wherein an organic polymer film acts as a reinforced support layer between the ultra-thin carbon film $(\sim 4 \mathrm{~nm})$ and copper mesh (Fig. 1a) (Regan et al. 2010; Warner et al. 2010; Kennedy et al. 1998). The organic polymer film is necessary for the HRTEM characterization of ultra-small nanomaterials, especially nanocrystalline materials. However, the organic polymer support layer can be dissolved by many general organic reagents (chloroform, methylbenzene, etc), which limits the sample preparation of oil-soluble nanomaterials (Kearns et al. 2006).

Correspondence: yuhaodeng@pku.edu.cn

Academy for Advanced Interdisciplinary Studies, Peking University, 209 Chengfu Road, Haidian District, Beijing 100871, China

\section{Materials and methods}

Methylbenzene (>99.7\%), naphthalene (>99.9\%) were obtained from Sinopharm Chemical Reagent Co., Ltd. (SCRC). TEM grid was obtained from Zhongjingkeyi Technology Co., Ltd. The synthesis of PbS nanocrystals is based on the described by Moreels et al. (2011). The naphthalene is heated to $110^{\circ} \mathrm{C}$ and cooled on glass slide to form naphthalene transfer layer. Optical images were acquired using optical microscope (Zeiss Axio Imager, A2m). All TEM, HAADF-STEM and HRTEM images were acquired using Tecnai F30 microscope operated at $300 \mathrm{kV}$.

\section{Results and discussion}

Figure $1 \mathrm{~b}$ and $\mathrm{c}$ show the TEM grid before and after treatment with methylbenzene respectively. The methylbenzene dissolved the organic polymer film and destroyed the skeleton structure of the TEM grid. The holes damaged by the organic solvent are circled with red dotted lines in Fig. 1c. Uneven and impaired grid will result in a difficult sample searching and sample drift, which will negatively affect the acquisition of highquality TEM images (Stinson-Bagby et al. 2018; Nair

\section{SpringerOpen}

(C) The Author(s). 2020 Open Access This article is licensed under a Creative Commons Attribution 4.0 International License, which permits use, sharing, adaptation, distribution and reproduction in any medium or format, as long as you give appropriate credit to the original author(s) and the source, provide a link to the Creative Commons licence, and indicate if changes were made. The images or other third party material in this article are included in the article's Creative Commons licence, unless indicated otherwise in a credit line to the material. If material is not included in the article's Creative Commons licence and your intended use is not permitted by statutory regulation or exceeds the permitted use, you will need to obtain permission directly from the copyright holder. To view a copy of this licence, visit http://creativecommons.org/licenses/by/4.0/. 

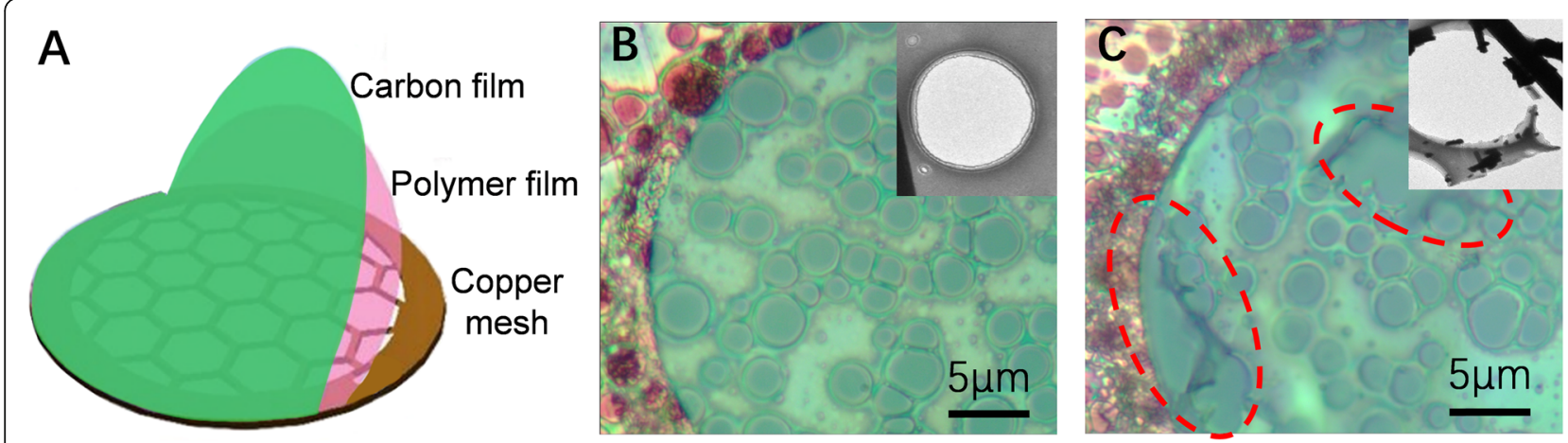

Fig. 1 Structure and property of TEM grid. a structure of TEM grid for nanomaterials, from top to bottom are carbon film, organic polymer film and copper mesh. $\mathbf{b}$ optical microscope and TEM images (inset) of unbroken TEM grid. c optical microscope and TEM images (inset) destroyed by methylbenzene
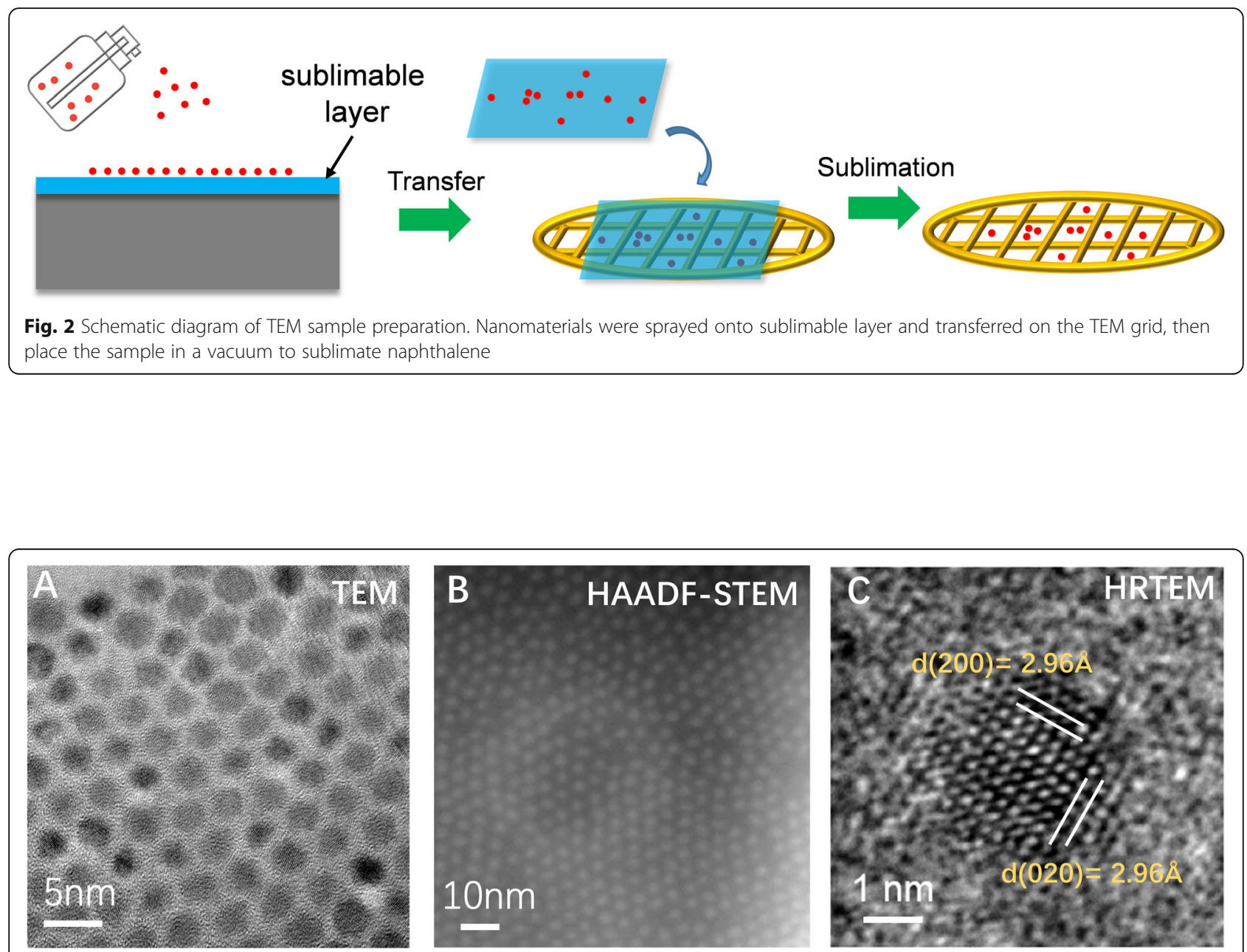

Fig. 3 TEM images of PbS nanocrystals sample via new preparation method. a TEM image at low magnification. $\mathbf{b}$ image of nanocrystals under HAADF-STEM mode. $\boldsymbol{c}$ image of HRTEM 
et al. 2010; Duchamp et al. 2014). Therefore, it is necessary to develop a method that can obtain high-quality TEM sample of oil-soluble nanomaterials and avoid damaging the organic polymer layer in TEM grid.

Here, a new method for nanomaterial sample preparation was designed by introduce sublimable naphthalene as the transition layer. First, naphthalene was deposited on the substrate and nanomaterials were sprayed onto naphthalene layer. Next, the side of the naphthalene layer with the nanomaterial was transferred to covered on the TEM grid, and then place the sample in a vacuum to sublimate naphthalene. The process is shown in Fig. 2.

Based on this method, we obtained a continuous large-scale highly-dispersed oil-soluble $\mathrm{PbS}$ nanocrystal sample and captured high-quality TEM (Fig. 3a), Highangle annular dark-field scanning transmission electron microscopy (HAADF-STEM) (Fig. 3b) and HRTEM (Fig. 3c) images. It proves that images with low background contrast, accurate material morphology, and clear lattice fringes can be obtained via this new sample preparation method.

\section{Conclusions}

This new method avoids the destruction of organic polymer layer in TEM grid by organic solvent and can obtain high-quality TEM samples in the characterization of oilsoluble nanomaterials. Our method not only can be used for the preparation of nanocrystals sample, but also can be applied to oil-soluble nanoclusters, nanosheets, nanowires and other materials. In addition to oil-soluble nanomaterials, other nanomaterials in corrosive solutions such as strong acids and bases can also be suitable for this method. This work enriches and improves the sample preparation technique in the field of TEM.

\section{Abbreviations}

TEM: Transmission electron microscopy; HRTEM: High resolution transmission electron microscopy; HAADF-STEM: High-angle annular dark-field scanning transmission electron microscopy; PbS: Lead sulfide

\section{Acknowledgments}

None.

\section{Author's contributions}

The author(s) read and approved the final manuscript.

\section{Funding}

None.

\section{Availability of data and materials}

Please contact the corresponding author for data availability.
Received: 4 September 2020 Accepted: 21 September 2020

Published online: 29 September 2020

\section{References}

Ayache J, Beaunier L, Boumendil J, et al. Sample preparation handbook for transmission electron microscopy: techniques[M]. Springer Science \& Business Media, (2010). https://www.springer.com/gp/book/9780387981819.

H.W. Cha, M.C. Kang, K. Shin, et al., Transmission electron microscopy specimen preparation of delicate materials using Tripod polisher. Appl. Microsc. 46(2), 110-115 (2016). https://doi.org/10.9729/AM.2016.46.2.110

M. Duchamp, Q. Xu, R.E. Dunin-Borkowski, Convenient preparation of high-quality specimens for annealing experiments in the transmission electron microscope. Microsc. Microanal. 20(6), 1638-1645 (2014). https://doi.org/10. 1017/S1431927614013476

G.J. Kearns, E.W. Foster, J.E. Hutchison, Substrates for direct imaging of chemically functionalized $\mathrm{SiO} 2$ surfaces by transmission electron microscopy. Anal. Chem. 78(1), 298-303 (2006). https://doi.org/10.1021/ac051459k

M.T. Kennedy, B.A. Korgel, H.G. Monbouquette, et al., Cryo-transmission electron microscopy confirms controlled synthesis of cadmium sulfide nanocrystals within lecithin vesicles. Chem. Mater. 10(8), 2116-2119 (1998). https://doi.org/ $10.1021 / \mathrm{cm} 970744 \mathrm{k}$

N.Y. Kim, G.H. Ryu, H.J. Park, et al., An improved specimen preparation of porous powder materials for transmission electron microscopy. Microsc. Microanal. 20(S3), 366-367 (2014). https://doi.org/10.1017/S1431927614003559

I. Moreels, Y. Justo, B. De Geyter, et al., Size-tunable, bright, and stable PbS quantum dots: A surface chemistry study. ACS Nano 5(3), 2004-2012 (2011). https://doi.org/10.1021/nn103050w

R.R. Nair, P. Blake, J.R. Blake, et al., Graphene as a transparent conductive support for studying biological molecules by transmission electron microscopy. Appl. Phys. Lett. 97(15), 153102 (2010). https://doi.org/10.1063/1.3492845

C.H. Park, H.W. Kim, I.J. Rhyu, et al., How to get well-preserved samples for transmission electron microscopy. Appl. Microsc. 46(4), 188-192 (2016). https://doi.org/10.9729/AM.2016.46.4.188

W. Regan, N. Alem, B. Alemán, et al., A direct transfer of layer-area graphene. Appl. Phys. Lett. 96(11), 113102 (2010). https://doi.org/10.1063/1.3337091

K.L. Stinson-Bagby, R. Roberts, E.J. Foster, Effective cellulose nanocrystal imaging using transmission electron microscopy. Carbohydr. Polym. 186, 429-438 (2018). https://doi.org/10.1016/j.carbpol.2018.01.054

J.H. Warner, M.H. Rummeli, A. Bachmatiuk, et al., Examining co-based nanocrystals on graphene using low-voltage aberration-corrected transmission electron microscopy. ACS Nano 4(1), 470-476 (2010). https://doi.org/10.1021/ $\mathrm{nn} 901371 \mathrm{k}$

\section{Publisher's Note}

Springer Nature remains neutral with regard to jurisdictional claims in published maps and institutional affiliations.

\section{Submit your manuscript to a SpringerOpen ${ }^{\circ}$ journal and benefit from:}

- Convenient online submission

- Rigorous peer review

- Open access: articles freely available online

High visibility within the field

- Retaining the copyright to your article

Submit your next manuscript at $>$ springeropen.com 